\title{
Driver's Big Brother: How Smartphones Can Increase Driver's Awareness
}

\author{
http://dx.doi.org/10.3991/ijim.v6i4.2188 \\ Ashraf Khali1 ${ }^{1}$ and Said Elnaffar ${ }^{2}$ \\ ${ }^{1}$ Abu Dhabi University, Al Ain, United Arab Emirates \\ ${ }^{2}$ UAE University, Al Ain, United Arab Emirates
}

\begin{abstract}
Using mobile devices while driving has been widely shown to pose serious safety risk. Various approaches aim at mitigating the risk of mobile devices while driving. Some initiatives have focused on increasing drivers' awareness of such potential risk. Along the same vein, we present a model, and its implementation, for assessing the degree of distraction that drivers experience while using phones during driving. Based on data collected during each driving session, the application gives statistics, such as keypad interaction, number and length of phone calls and the driving speed, to the driver. This should increase the awareness of drivers by helping them understand how much distraction they got involved with during driving and how much hazardous they can be to themselves and to others. Our experiments with drivers demonstrated the potential of the application at highlighting risky behavior, promoting awareness and motivating better driving.
\end{abstract}

Index Terms-Driver Riskometer, driver distraction, motion classifier, risk assessment.

\section{INTRODUCTION AND MOTIVATION}

Several research studies have addressed the risk of using mobile phones while driving [1] [2]. Virginia Tech Transportation Institute (VTTI) provides a clear picture of driver distraction and cell phone use under real world driving conditions [1]. In [1], it was shown that those who text while driving are 23 times more likely to crash or nearly crash than those who are not distracted. Comparatively, those who dial phone numbers are 2.8 times more likely to crash or nearly crash while those who talk on a cell phone are about 1.3 times as likely to crash.

Despite the general consensus that mobile phones impose a driving safety risk, there is neither consensus on the magnitude of the risk nor on the best approach to lower it. Many countries have issued legislation to ban cell phone use while driving but a new study by the National Institute for Highway Safety claims that such banning unfortunately has no effect on accident rates [3].

Another approach to fight this problem is educating mobile users and improving their awareness of the issue because studies have found that people underestimate the risk or have mistaken information about mobile use while driving. For example, a study [2] found that $87.7 \%$ of drivers believe that hands-free device makes talking on phone while driving is a safer practice while, in fact, empirical experiments have shown that driving while using a hands-free cellular device is not safer than the direct usage of the handset [1].

In this paper, we present Driver Riskometer, a mobile system that aims at promoting safe driving behavior by measuring drivers' distractions caused by mobile use while driving.

It seamlessly runs as a background service and gets awakened automatically, without any intervention from the user (driver) as it autonomously detects if the user is in a driving mode. Once the driving mode is recognized, the Driver Riskometer kicks off by collecting usage statistics for the mobile device in order to assess how risky the concerned driver is. It does so by tracking three measures: speed of the vehicle, number of phone calls received or issued, and the number of presses/interactions with on the keypad especially for the purpose of texting and emailing while driving.

The system shows these statistics to the user at the end of the road trip so that we do not add one extra distraction to the driver. The application does not record any private information such as the GPS locations, phone numbers, or text messages, making it privacy-friendly.

The rest of the paper is structured as follows. The first section covers the some related work. The next section describes the system design and implementation. The User Study section discusses our experiments, followed by a section that highlights the most important results and observations. The last section concludes the paper and sketches future work.

\section{RELATED WORK}

Our work leverages previous research on driver distractions and changing driver behavior. Various studies have been conducted to measure driver distraction using driving simulators [4], and instrumented vehicles on the road [1] but the purpose of these studies was ultimately collecting collective data rather than providing each individual with information about her own driving behavior.

WatchMe [5]is a system that aims at choosing the best way to communicate with buddies. WatchMe relies on GPS trace analysis to determine whether a person is walking or driving while Driver Riskometer uses the mobile accelerometer sensor to determine when the user starts driving because the accelerometer is a very cheapest sensor in terms of energy consumption [6].

There exist a few off-the-shelf mobile applications that aim at changing driver behavior. For example, Bliss Trek [7] and Nissan's iPhone eco-driving application [8] both encourage drivers to follow eco-driving practices by measuring the car acceleration and how well the driver keeps a constant speed while cruising. Also, DriveSafe.ly [9] is a free mobile application that reads text messages and emails aloud in real time and automatically responds without users touching their mobile phone. ZoomSafer 
[10] is another mobile software that automatically detects when your car is in motion and locks the phone's screen and keypad, which prevents you from checking your mail or sending messages. It also can be set to auto-respond to incoming messages ("I'm on the road and will reply later") and simplify hands-free call reception by announcing who is calling.

Another system which aims at changing driver behavior is First UK Bus [11], for UK's largest bus operator. The system assesses drivers' performance by analyzing how well they perform maneuvers such as cornering and breaking using its onboard motion detector, GPS receiver and computer. Drivers are also expected to learn from the system, which provides them with instantaneous feedback on how well they are performing, using an LED display on the dashboard that flashes red, yellow or green The idea is that drivers will be motivated to keep the light green and in the process they will learn to improve their driving skills.

\section{SYSTEM DESIGN AND IMPLEMENTATION}

Figure 1 shows the general architecture of the Riskometer system, which happened to be implemented on the Nokia N95 (8 GB) smartphone. It consists of two main modules: Analytical module and the Monitoring module that runs as a system service. The first module is coded in Java (J2ME) for the sake of platform portability, while the Monitoring module had to be implemented in $\mathrm{C}++$ in order to access the low-level system resources such as sensors and logs. The latter module captures system data, such as the number of keypad clicks or screen touches, and passes it on to the Analytical module for manipulation.

\section{A. The Monitoring Module}

It is a Symbian based service that captures system interactions with the low-level system resources and passes on these statistics to the Analytical module (J2ME) through socket connections. These systems resources include: the call log, keypad, accelerometer sensor and the daemons watcher.

The monitoring module undertakes the following tasks:

- Detecting incoming/outgoing calls and their duration

- Detecting keypad interactions and their duration

- Polling the accelerometer sensor readings, and

- Start the other daemons when the user starts Driver Riskometer midlet as well as restarting any daemons that exit while the midlet is still running.

\section{B. The Analytical Module}

This module is implemented using a J2ME midlet which consists of several components: call log client, keypad client, accelerometer client, watcher client, GPS client, local storage client and the GUI client. These components work in collaboration with the Monitoring module in order to obtain the required system data and usage statistics.

\section{How the Driver Riskometer Works}

Many precautions were taken into account when designing the Driver Riskometer in order to make it a lightweight utility. Upon installing this application, the Monitoring module is the only that keeps running in the background as a system service. However, all of its 8

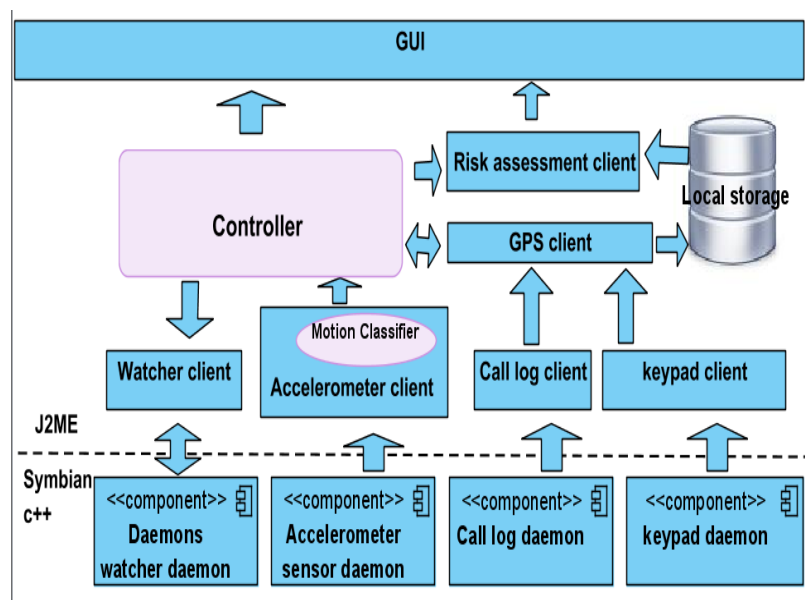

Figure 1. Architecture of the Driver Riskometer system

components remain inactive except the accelerometer sensor one, which is virtually turned on all the time on all devices by default as it knowingly does not consume energy.

The Monitoring module continually collects a stream of data from the accelerometer and passes it on to the accelerometer client inside the Analytical module via socket connections. The accelerometer client works as a motion classifier in order to detect whether the user is engaged in a driving session.

Given the intrinsic limited resources, computation and storage, of smartphones, we adopted a simple working algorithm for recognizing the driving motion. The motion classifier computes the slope of the last 120 accelerometer readings, the sum of the absolute value of the slopes, and number of peaks of the slops readings. We empirically found that the combination of the sum of the absolute value of the slopes, and number of peaks of the slopes readings uniquely identify car motion.

Upon detecting 9 successive car motions, which takes on average 27 seconds, a new driving session (journey) is declared. Subsequently, the GPS client is triggered in order to sense the speed of the vehicle, the accelerometer client is suspended, the call log and keypad clients are watched in order to intercept any user interactions with them.

During the journey the GPS client collects samples from vehicle speeds and stores them locally and computes some simple statistics such as the average speed and the maximum one reached.

At the end of the journey, which is identified when the vehicle's speed drops to $7 \mathrm{~km} / \mathrm{h}$ or less for 4 minutes, the GPS client signals the call log key presses client to stop collecting usage data. Using the GUI client, statistics such as number of calls received and made, their duration, number of key presses, etc., collected throughout the journey are displayed to the user upon finishing the driving session as shown in Figure 2.

\section{USER STUDY}

We conducted a preliminary user study to bring Driver Riskometer to the attention of potential users and solicit their feedback. We gave the participants a pre-study questionnaire to collect data about their current driving 


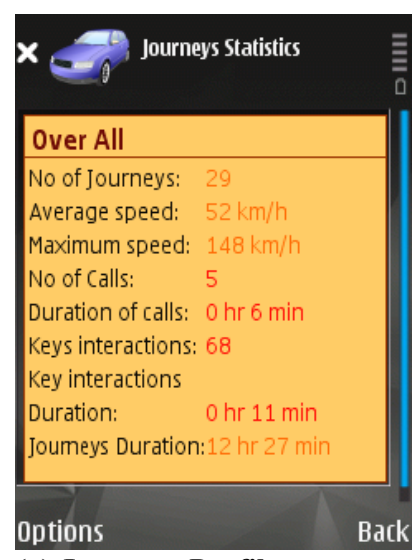

(a) Journeys Profile

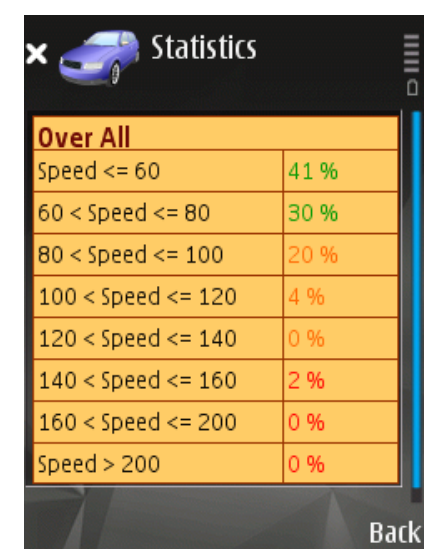

(b) Speed Profile
Figure 2. Some of the statistics generated by the Driver Riskometer

behavior then, and using post-study semi-structured interviews, we asked them to use Driver Riskometer and provide feedback about their user experience.

The study was conducted over a two weeks period and it involved 12 participants, 5 males and 7 females. The set of candidates consisted of 6 undergraduate students, 3 graduate student, 1 master degree holder staff, and 2 professors. The average age of the participants is 26 with a standard deviation of 6 years. Ten of the participants were warned for exceeding speed limit and 10 wore seatbelt all the time. When we asked them how often do you pull off the road to use the cell phone, 7 of them answered 'Never', 4 answered 'Rarely' and one responded 'Sometimes'.

The purpose of the post-study interviews was to find out the effect of using Driver Riskometer on changes in participants' perception and knowledge about their driving behavior. We asked open-ended questions in five categories: awareness, ease of use, utility, information sharing, and design aspects. The results described next.

\section{RESULTS}

The purpose of the post-study interviews was to find out the effect of using Driver Riskometer on changes in participants' perception and knowledge about their driving behavior. We asked open-ended questions in five categories: awareness, ease of use, utility, information sharing, and design aspects. A summary of the results follows.

\section{A. Awareness}

Participant had very interesting comments about how Driver Riskmeter highlighted or revealed some of their driving behavior.

One participant said "I noticed that my driving behavior changed when I drive with my family. Also, my wife is becoming aware of my driving behavior as she warns me from driving dangerously".

Another participant said "I was not aware that I keep tampering with my mobile while driving but the application drew my attention to that risky habit."

One participant said "Even before I see the application results, I notice that I was more conscious of receiving a call knowing that the application is monitoring me".

\section{B. Ease of Use}

All participants agreed on the simplicity and ease of use of driver Riskometer.

One participant said "The application recognize that I'm in the car without my intervention but I also want it to recognize if I'm the driver or not"

\section{Utility}

Nine participants used the application in every journey they took, 11 of them said they will continue using it after the study because they like the different type of information it provides (see Figure 2). Only 1 participant said "I will not use the application afterward. I consider myself a safe driver and I would not make a call while driving unless it is emergency."

Participants were very pleased with the detailed information Driver Riskometer provides. On participant said "Speed details and journey details are my two favorite views" Anther participant said "I check my maximum speed after long journeys to see if I have exceeded speed limit and whether I had been caught by radar"

\section{Sharing Experiences among Users}

All participants said that they have shared their driving information with the spouse, relatives and friends. Participant also mentioned that some of their friends may mind sharing their driving behavior with their parents. One participant said "Although I have shared my driving information with my father and brother I will not share it with my husband because he will deny driving if I exceeded certain speed". One participant suggested adding peer communication tool which allow users to share with each other their driving information.

\section{E. Design Aspects}

Seven participants asked us to add sound alarm when the driver reaches certain speed. Another participant suggested creating profile for different drivers in the same mobile while a third participant suggested asking the user if he or she is the current driver of the vehicle instead of assuming that the user is always the driver. We found this to be a serious design problem that we need to address. Three participants suggested using calendar view to display historic information.

\section{CONCLUSION}

The main aim of Driver Riskometer application is to raise the drivers' awareness of their driving behavior by tracking watching their cell phone usage and speeding pattern. We argued that such information and statistics will have a positive effect on the safety of their driving profile.

The preliminary user study we conducted showed that users are finding the application useful, engaging, and above all effective in changing their driving behavior. Long term behavioral change is hard to gauge in such a limited user study but another more comprehensive user study will be conducted in the near future for this purpose.

One of the main features of our application is that it does not require any additional hardware components and it is designed to be easily portable to other smart phone platforms. In addition to the self-assessment, Driver Riskometer is suitable for parental monitoring where 


\section{SHORT PAPER}

\section{DRIVER's BIG BROTHER: HOW SMARTPHONES CAN INCREASE DRIVER'S AWARENESS}

parents can use it to monitor the manners of their teenagers on the road. Other potential application domains in which this system can be used is businesses that have fleets or school buses where drivers need to be extra disciplined.

In the future we aim to add the capability of setting goals and measuring improvements. Another issue we will be exploring is the effect of adding a social component to the application so users can share driving patterns with friends or family members. Furthermore, we are investigating the possibility of assigning a risk factor that combines the three different readings (speed, talking and texting) and provide the user with one risk level in addition to the ability to view the complete data if desired. To that end, the user can get an immediate feedback about his or her driving behavior without the need of reading all the statistics. The risk level can be calculated using fuzzy logic and calibrated by a driving risk expert.

\section{REFERENCES}

[1] S. Box, "New Data from VTTI Provides Insight into Cell Phone Use and Driving Distraction," Virginia Tech Transportation Institute, 2009.

[2] C. Jane, F. H. Herman and W. W. H., "Cell Phone Use While Driving: Results of a Statewide Survey.," TRB 2003 Annual Meeting CD-Rom, 2003.

[3] "Laws banning cellphone use while driving fail to reduce crashes, new insurance data indicate,", 2010. [Online]. Available: http://www.iihs.org/news/rss/pr012910.html [Accessed 2010].
[4] H. Alm and L. Nilsson, "The effects of a mobile telephone task on driver behaviour in a car following situation," Accident Analysis and Prevention, pp. 707-715, 1995. http://dx.doi.org/10.1016/ 0001-4575(95)00026-V

[5] N. Marmasse, C. Schmandt and D. Spectre., "WatchMe: Communication and Awareness Between Members of a Closelyknit Group," 2004.

[6] B. A. A. P. H. T. Fehmi, "Less is More: Energy-Efficient Mobile Sensing with SenseLess.," MobiHeld2009, 2009.

[7] "Bliss Trek," 2010. [Online]. http://www.blisstrek.com/. [Accessed 2010].

[8] "Nissan's iPhone eco-driving application," 2010. [Online]. http://www.macworld.com/article/143183/2009/10/nissan iphone drivingapp.html. [Accessed 2010].

[9] "DriveSafe.ly," 2010. [Online]. http://www.DriveSafe.ly. [Accessed 2010].

[10] "ZoomSafer," 2010. [Online]. http://zoomsafer.com/. [Accessed 2010].

[11] "First UK Bus system," 2010. [Online]. http://news.zdnet.co.uk/ software $/ 0,1000000121,39706539,00 . \mathrm{htm}$. [Accessed 2010].

\section{AUTHORS}

A. Khalil is an Assistant Professor at the College of Information Technology, Abu Dhabi University, Al Ain, UAE (e-mail: ashraf.khalil@adu.ac.ae).

S. EInaffar is an Associate Professor at the College of Information Technology, UAE University, Al Ain, UAE (e-mail: elnaffar@uaeu.ac.ae).

Received 16 July 2012. Published as resubmitted bx'y the authors 23 September 2012. 